\title{
Outcome of External Ventricular Drainage according to the Operating Place: the Intensive Care Unit versus Operating Room
}

\author{
Si On Kim, M.D. ${ }^{1}$, Won Jun Song, M.D. ${ }^{1}$, Yu Sam Won, M.D. ${ }^{2}$, Jae Young Yang, M.D. ${ }^{2}$, and Chun Sik Choi, M.D. ${ }^{2}$ \\ ${ }^{1}$ Department of Critical Care Medicine, ${ }^{2}$ Department of Neurosurgery, Sungkyunkwan University, Kangbuk Samsung Hospital, Seoul, Korea
}

Background: External ventricular drainage (EVD) is an important procedure for draining excessive cerebrospinal fluid (CSF) and monitoring intracranial pressure. Generally, EVD is performed in the operating room (OR) under aseptic conditions. However, in emergency circumstances, the operation may be performed in the intensive care unit (ICU) to save neuro-critical time and to avoid the unnecessary transfer of patients. In this study, we retrospectively analyzed the risk of EVD-induced CNS infections and their outcomes according to the operating place (ICU versus OR). In addition, we compared mortalities as well as hospital and ICU days between the CNS infection and non-CNS infection groups.

Methods: We reviewed medical records, laboratory data and radiographic images of patients who had received EVD operations between January, 2013 and March, 2015.

Results: A total of 75 patients (45 men and 30 women, mean age: $58.7 \pm 15.6$ years) were enrolled in this study. An average of 1.4 catheters were used for each patient and the mean period of the indwelling catheter was $7.5 \pm 5.0$ days. Twenty-six patients were included in the ICU group, and EVD-induced CNS infection had occurred in 3 (11.5\%) patients. For the OR group, forty-nine patients were included and EVD-induced CNS infection had occurred in 7 (14.3\%) patients. The EVD-induced CNS infection of the ICU group did not increase above that of the OR group. The ICU days and mortality rate were higher in the CNS infection group compared to the non-CNS infection group. The period of the indwelling EVD catheter and the number of inserted EVD catheters were both higher in the CNS infection group.

Conclusions: If the aseptic protocols and barrier precautions are strictly kept, EVD in the ICU does not have a higher risk of CNS infections compared to the OR. In addition, EVD in the ICU can decrease the hospital and ICU days by saving neuro-critical time and avoiding the unnecessary transfer of patients. Therefore, when neurosurgeons decide upon the operating place for EVD, they should consider the benefits of ICU operation and be cautious of EVD-induced CNS infection.

Key Words: catheter-related infections; CNS infection; intensive care unit; ventriculostomy.

\section{Introduction}

External ventricular drainage (EVD), also known as ventriculostomy, is the most common and effective procedure in neurointensive care.[1-4] EVD is performed for various purposes including: reducing and monitoring the intracranial pressure (ICP), drainage of excessive cerebrospinal fluid (CSF) and removal of intraventricular hematoma. This procedure is generally regarded as safe and minimally harmful, therefore, in emer-

Received on August 22, 2015 Revised on February 1, 2016

Accepted on February 21, 2016

Correspondence to: Si On Kim, Department of Critical Care Medicine, Kangbuk Samsung Hospital, 29 Saemunan-ro, Jongno-gu, Seoul 03181, Korea

Tel: +82-2-2001-2158, Fax: +82-2-2001-2157

E-mail: kkimsion@hanmail.net

*No potential conflict of interest relevant to this article was reported. gency circumstances, EVD is performed in the intensive care unit (ICU).[5] Although EVD is very useful and essential in neurosurgical practice, it still has considerable complications. Specifically, catheter-related infections, cerebral hemorrhages, improper catheter insertions and brain parenchymal injuries, 
could occur. Of these complications, catheter-related infections can cause ventriculitis, meningitis and brain abscesses, and can thereby, result in severe neurological damages.[1,4,6-9]

The purpose of the current study is to compare the various factors associated with EVD-induced central nervous system (CNS) infection, according to the operating place, specifically ICU versus operating room (OR), and to evaluate the outcomes of EVD-induced CNS infections.

\section{Materials and Methods}

Retrospectively, we reviewed all patients who underwent EVD between January, 2013 and March, 2015 at our single institution. The patients that were aged 18 years or over, and had an EVD catheter for more than 48 hours were included, whilst the patients who previously had CNS infections were excluded. We reviewed medical records, laboratory data and radiographic images of all enrolled patients, and collected data on age, sex, diagnosis, operating place, numbers of inserted EVD catheters, indwelling period, mortality rate, hospital days, and ICU days.

\section{1) The definition of infection}

We defined "CNS infection" based on the published standards from the Center for Disease Control and Prevention and many other studies.[1,4,5,9-11] We categorized EVDinduced CNS infections into "Confirmed CNS infections" and "Suspected CNS infections".

(1) Confirmed CNS infection: The presence of pathogens in the CSF or catheter tip cultures.

(2) Suspected CNS infection: The absence of pathogens in the CSF or catheter tip cultures, but presence of fever (body temperature $\geq 37.4^{\circ} \mathrm{C}$ ) and meningeal irritation signs with gradual increase of white blood cell/red blood cell and Protein/Glucose ratios in serial CSF tests.

We checked baseline CSF test and culture when the EVD was first performed. Except for this initial test, we did not check CSF tests and cultures periodically. However, when the patients were suspected of having a CNS infection (fever of unknown origin, or meningeal irritation signs), we took the CSF tests and cultures every other day. The CSF tests and cultures were collected at the three-way taps of the EVD drainage system, using aseptic protocol. After EVD catheter removal, the catheter tips were sent to the microbiological laboratory, to confirm the CNS infection.

\section{2) Operations and post-operative care}

The operations were performed by senior residents who had undergone more than 3 years of training. Aseptic protocols and surgical techniques were applied equally in both places (ICU or OR). Our aseptic protocol for EVD included use of hair clip, betadine scrubs and maximal barrier precautions (use of surgical caps, masks, sterile gowns, gloves and drapes). After betadine painting and aseptic draping on the operating field, a small burr hole was drilled at the Kocker's point (mid-pupillary line in the sagittal plane and $1 \mathrm{~cm}$ anterior of the coronal suture). A twist-drill was used in the ICU, while an electrical-drill was used in the OR. An uncoated EVD catheter (neither antibiotic-impregnated nor silver-coated EVD catheters) was carefully inserted 5.5-7 $\mathrm{cm}$ through the burr hole, and a closed drainage system was connected. Post-operative care was managed at the ICU during the period of indwelling EVD catheters. All patients received antibiotic prophylaxis with first or third generation cephalosporin until the EVD catheters were removed, and sterile dressings were applied every other day.

\section{3) Statistical analysis}

Numerical data were presented in terms of the mean range, and tested for statistical significance using the Student t-test. Categorical data were indicated as frequencies and percentages, and then compared using the Pearson Chisquare test, or Fisher's exact test. The difference was considered statistically significant when the $\mathrm{p}$ value was less than 0.05. All statistical analyses were conducted using IBM SPSS Statistics version 22 (SPSS, Inc., Chicago, IL, USA).

\section{Results}

Seventy-five patients were included in this study, and the mean age of the patients was $58.7 \pm 15.6$ years old. The initial diagnoses of the patients were $55(73.3 \%)$ vascular diseases, $9(12.0 \%)$ head traumas, $6(8.0 \%)$ brain tumors, $4(5.3 \%)$ cerebral infarctions, and 1 (1.3\%) Arnold-Chiari malformation. As well as this, $16(21.3 \%)$ hydrocephalus cases were concurrently detected, and the initial Glasgow 
coma scale (GCS) of the patients was $8.9 \pm 3.6$. Vascular diseases included, hypertensive intracerebral hemorrhage, subarachnoid hemorrhage, rupture of the arteriovenous malformation, Moyamoya disease and intraventricular hematoma. Head traumas included traumatic subdural hemorrhage, traumatic subarachnoid hemorrhage, and intracerebral contusion. One point four catheters were used for each of the 75 patients, and the mean period of an indwelling EVD catheter was $7.5 \pm 5.0$ days. Of the 75 patients, 10 (13.3\%) patients were diagnosed with EVD-induced CNS infection and $27(36.0 \%)$ patients expired due to the initial diagnosis (Table 1).

Among the 75 patients, $26(34.7 \%)$ patients were operated on in the ICU and $49(65.3 \%)$ patients were operated on in the OR. Between the ICU and OR groups, sex, age, causative disease, and initial hydrocephalus were not shown sta-

Table 1. Demographic and clinical characteristics of the patients $(\mathrm{n}=75)$

\begin{tabular}{lc}
\hline Variables & Values \\
\hline Male:Female & $45: 30$ \\
\hline Age (years) & $58.7 \pm 15.6$ \\
\hline Cause & \\
\hline Vascular dz. & $55(73.3 \%)$ \\
\hline Trauma & $9(12.0 \%)$ \\
\hline Tumor & $6(8.0 \%)$ \\
\hline Infarction & $4(5.3 \%)$ \\
\hline Arnold-Chiari malformation & $1(1.3 \%)$ \\
\hline Hydrocephalus & $16(21.3 \%)$ \\
\hline GCS & $8.9 \pm 3.6$ \\
\hline Total number of inserted EVD catheters & 101 \\
\hline Number of inserted EVD catheters per person & $1.4 \pm 0.6$ \\
\hline Period of indwelling EVD catheter (days) & $7.5 \pm 5.0$ \\
\hline Operating place (ICU:OR) & $26: 49$ \\
\hline EVD-induced CNS infection & $10(13.3 \%)$ \\
\hline Mortality & $27(36 \%)$ \\
\hline Hospital days & $52.9 \pm 60.0$ \\
\hline ICU days & $21.7 \pm 26.0$ \\
\hline Survivors & $10.4 \pm 3.1$ \\
\hline GCS & $67.1 \pm 61.4$ \\
\hline Cospital days & $22.8 \pm 29.4$ \\
\hline . & \\
\hline
\end{tabular}

Dz.: diseases, GCS: Glasgow coma scale, EVD: external ventricular drainage, ICU: intensive care unit, OR: operating room, CNS: central nervous system. tistical significance. The infection rate of the ICU group was slightly lower than that of the OR group, but without statistical significance. Three patients $(11.5 \%)$ of the ICU group, and seven patients $(14.3 \%)$ of the OR group were diagnosed with EVD-induced CNS infection $(\mathrm{p}=0.739)$. And in both groups, Acinetobacter baumannii with multi-drug resistance (MDR) were grown in CSF cultures (Table 2).

Because the initial GCS of the ICU group was significantly lower than that of the OR group, the patients of the ICU group were expired early. As a result, in the ICU group, the mortality rate was high, and hospital and ICU days were low. For excluding the effects of the early mortality, we performed subgroup analysis with the surviving patients. In the subgroup analysis, although the initial neurological states (GCS) of the ICU group were significantly worse than those of the OR group (ICU group: $9.0 \pm 4.0$, OR group: $11.1 \pm$ $2.4, \mathrm{p}=0.049$ ), however, hospital and ICU days of the ICU group were all decreased, when compared to those of the OR group (Table 2).

Between the CNS infection and non-CNS infection group, there was no statistical significance, except the ICU days. The ICU days were significantly higher in the CNS infection group (infection group: $32.9 \pm 22.8$, non-infection group: $20.0 \pm 26.2, p=0.014)$. In the CNS infection group, EVD was performed more frequently and maintained longer period without significance. And CNS infection group tended to occur in patients who had a hydrocephalus and low GCS (Table 3).

\section{Discussion}

As an ICP monitoring device and as a direct portal for the removal of CSF or hematoma, EVD plays an important role in a broad spectrum of CNS diseases. Moreover, the surgical technique of EVD is relatively safe and minimally harmful, therefore, in emergency circumstances, the operation can be performed in the ICU. In spite of these advantages, EVD does have considerable complications such as, catheter-related infection, cerebral hemorrhage, improper insertion, and brain parenchymal injury. Of these complications, EVD-induced CNS infection especially, has been an unresolved problem since its introduction.[1,4,6-9] Despite various precautions for preventing CNS infection, up to 
Table 2. Clinical results of the ICU group and OR group

\begin{tabular}{|c|c|c|c|}
\hline Variables & ICU $(n=26)$ & $\mathrm{OR}(\mathrm{n}=49)$ & $\mathrm{p}$-value \\
\hline Male:Female & $15: 11$ & $30: 19$ & 0.766 \\
\hline Age (years) & $57.9 \pm 16.6$ & $59.0 \pm 15.2$ & 0.770 \\
\hline Cause & & & 0.264 \\
\hline Vascular dz. & $17(65.4 \%)$ & $38(77.6 \%)$ & \\
\hline Trauma & $3(11.5 \%)$ & $6(12.2 \%)$ & \\
\hline Tumor & $4(15.4 \%)$ & $2(4.1 \%)$ & \\
\hline Infarction & $1(3.8 \%)$ & $3(6.1 \%)$ & \\
\hline Arnold-Chiari malformation & $1(3.8 \%)$ & $0(0 \%)$ & \\
\hline Hydrocephalus & $5(19.2 \%)$ & $11(22.4 \%)$ & 0.495 \\
\hline GCS & $7.2 \pm 3.9$ & $9.9 \pm 3.2$ & $0.002^{*}$ \\
\hline EVD-induced CNS infection & $3(11.5 \%)$ & $7(14.3 \%)$ & 0.739 \\
\hline Confirmed CNS infection & $3(11.5 \%)$ & $2(4.1 \%)$ & \\
\hline Suspected CNS infection & $0(0 \%)$ & $5(10.2 \%)$ & \\
\hline Pathogens & $\begin{array}{c}\text { Acinetobacter baumannii } 2 \\
\text { Candida glabrata }\end{array}$ & $\begin{array}{l}\text { Acinetobacter baumannii } \\
\text { Staphylococcus hominis }\end{array}$ & \\
\hline Mortality & $11(42.3 \%)$ & $16(32.7 \%)$ & 0.407 \\
\hline Hospital days & $36.0 \pm 43.2$ & $61.9 \pm 65.9$ & $0.007^{*}$ \\
\hline ICU days & $15.8 \pm 15.4$ & $25.0 \pm 29.9$ & 0.085 \\
\hline Survivors & $15(57.7 \%)$ & $33(67.3 \%)$ & \\
\hline GCS & $9.0 \pm 4.0$ & $11.1 \pm 2.4$ & $0.049^{*}$ \\
\hline Hospital days & $54.2 \pm 49.7$ & $73.0 \pm 66.0$ & 0.339 \\
\hline ICU days & $19.1 \pm 19.2$ & $24.5 \pm 33.3$ & 0.486 \\
\hline
\end{tabular}

ICU: intensive care unit, OR: operating room, Dz:: diseases, GCS: Glasgow coma scale, EVD: external ventricular drainage, CNS: central nervous system.

Table 3. Clinical differences between CNS infection and non-CNS infection

\begin{tabular}{lccc}
\hline \multicolumn{1}{c}{ Variable } & CNS infection $(\mathrm{n}=10)$ & Non-CNS infection $(\mathrm{n}=65)$ & $\mathrm{p}$-value \\
\hline Male:Female & $6: 4$ & $39: 26$ & 1.0 \\
Age (year) & $60.1 \pm 15.1$ & $58.4 \pm 15.8$ & 0.755 \\
Number of inserted EVD catheters per person (catheter/person) & $1.6 \pm 0.8$ & $1.3 \pm 0.5$ & 0.290 \\
Period of indwelling EVD catheter (days) & $10.8 \pm 9.26$ & $7.0 \pm 3.9$ & 0.290 \\
Hydrocephalus & $3(30 \%)$ & $13(20 \%)$ & 0.563 \\
GCS & $7.0 \pm 2.7$ & $9.2 \pm 3.7$ & 0.069 \\
Mortality & $6(60 \%)$ & $21(32.3 \%)$ & 0.154 \\
Hospital days & $51.8 \pm 42.7$ & $53.1 \pm 62.5$ & 0.436 \\
ICU days & $32.9 \pm 22.8$ & $20.0 \pm 26.2$ & $0.014^{*}$ \\
\hline
\end{tabular}

CNS: central nervous system, EVD: external ventricular drainage, GCS: Glasgow coma scale, ICU: intensive care unit.

$27 \%$ of patients with EVD suffer from CNS infections and the mortality rate of such patients increases 2-fold compared with patients without infections.[4,12] Aside from mortality rate, EVD-induced CNS infections can lead to other serious problems, including seizures, cognitive dysfunction, de- creased mentality, neurological deficits, prolonged number of hospital days, and expensive hospital costs.

EVD-induced CNS infections mostly occur by coagulasenegative staphylococcus (50-90\%) and Staphylococcus aureus (13-27\%) which are part of normal skin flora.[10,13,14] 
These microorganisms fenestrate into the ventricles through various routes, for example, contaminated catheters, surgical site infections, frequent catheter manipulation, prolonged catheter indwelling and through the drainage system itself. Alongside this, numerous factors also influence EVDinduced CNS infections indirectly through risk factors such as, previous neurosurgical operations, underlying disease and systemic infections.[15-17]

In this study, we have analyzed various factors of both the ICU and OR groups (Table 2). The basic characteristics such as, sex, age, causative disease, and hydrocephalus were not different between the two groups. However, the initial GCS of the ICU group was significantly lower than that of the OR group (ICU group: 7.2 \pm 3.9 , OR group: $9.9 \pm 3.2, \mathrm{p}=0.002$ ) When the neurological state of the patient was considered poor, we preferred to operate in the ICU to save neuro-critical time, because the preparation time in the OR is usually longer than that in the ICU. As a result, the patients who had been admitted with poor neurological states tended to be included in the ICU group. This difference influenced the mortality rate, as well as the hospital and ICU days, because the patients with lower initial GCS were expired earlier. Actually, in the ICU group, the mortality rate was higher, and the hospital and ICU days were relatively lower when compared to the OR group. To minimize this selection bias of operating place, we reanalyzed the data of only the surviving patients (48 patients). In subgroup analysis, although the neurological state of the ICU group was poorer (ICU group: $9.0 \pm 4.0$, OR group: $11.1 \pm 2.4, \mathrm{p}=0.049)$, but the hospital and ICU days were indeed lower in the surviving patients of the ICU group. This allows us to presume that early operation in the ICU could lead better outcomes for surviving patients. In neurosurgical practice, various factors can influence the outcomes of patients. Among them, the time delay prior to the operation is directly connected with the poor prognosis and maintenance of stable vital sign in acute phase is also crucial point. In usual hospital system, EVD in the OR needs more time than that in the ICU, because many considerations such as, medical staffs (anesthesiologist, assisting nurse), patient's condition, hospital process and operating room, are involved in that operation. While EVD in the ICU reduces those considerations, thereby, the time delay prior to the operation can be shortened. Furthermore, EVD in the ICU does not need patients to transfer to the OR. During the transfer, the patients cannot be managed properly, and changing bed (to transfer bed or operating table) can also affect adversely in acute phase. In the course of transfer and changing bed, unexpected situation such as, abrupt hypertension/hypotension, desaturation, airway problem, and fluid disconnection can be possible to the patients, and proper managements may not be applied immediately. Considering these adverse effects, we suppose that EVD in the ICU enables the patients to undergo an early operation and avoid unnecessary patient transfer, and that lead to shortening of hospital and ICU days (Table 2).

When we consider the operation in the ICU, the possibility of EVD-induced CNS infection is a major concern. It is a generally-accepted concept that the ICU operation has a higher potential risk of CNS infection, than the OR operation. However, in our study, the infection rate of the ICU group was not higher than that of the OR group (ICU group: $11.5 \%$, OR group: $14.3 \%, \mathrm{p}=0.739$ ). When we operate in the ICU, aseptic protocols were strictly adhered to, and the same equipment was used as in the OR, except drill type. Thereby, the infection rate slightly decreased in the ICU group. In previous study, Foreman et al. also reported that the infection rate of the ICU group was not different than that of the OR group.[5] We expect that if the operators notice a CNS infection, take appropriate precautions and keep to aseptic protocols, then the infection rates of ICU operations would not differ from those of the OR operations (Table 2).

Alongside this, we compared the clinical differences between infection and non-infection groups (Table 3). Although many other factors such as, initial diagnosis, age and underlying diseases could influence the mortality rate, as well as hospital and ICU days, in our study, EVD-induced CNS infection increased the ICU days and mortality rate. In particular, the ICU days of infection group were significantly higher than that of the non-infection group (infection group: $32.9 \pm 22.8$, non-infection group: $20.0 \pm 26.2, \mathrm{p}=$ 0.014). In previous studies, $[5-7,10,11]$ it was reported that the EVD-induced CNS infection increased mortality rate 2-fold and lead to poor neurological outcomes. CNS infection impedes neurological recovery, and thereby, the numbers of hospital and ICU days were prolonged.

The frequency of EVD and indwelling periods were slightly higher in the CNS infection group, but there were no statistical significance. As other catheter-related infec- 
tions are influenced by the frequency of procedure and indwelling period, we presume that in these cases frequent operations and longer indwelling periods had led to EVDinduced CNS infections (Table 3).

One of the serious concerns in this study was the prevalence of MDR microorganisms as a pathogen of CNS infection. CSF cultures or catheter tip cultures were positive in only $5(6.7 \%)$ patients. However, of these five patients, Acinetobacter baumannii which does not respond to conventional antibiotics had been grown in 3 patients. MDR microorganisms are more prevalent and widespread in current hospitals, including in the ICU and OR.[3] We suspect that these flourishes of MDR microorganisms influence the pathogens of EVD-induced CNS infections. Moreover, the antibiotics which could fenestrate the blood-brain barrier are very limited, and the CSF concentration of antibiotics after intravenous administration is very low. Accordingly, stronger antibiotics have to be administered for eradicating MDR microorganisms, and the patients endure the serious side effects of these antibiotics. Consequently, patients infected by MDR microorganisms exhibit more severe prognosis.[12]

There are some limitations to our study. Firstly, our study population was small, and the standard deviations of the hospital and ICU days were wide. As a result, statistical significance was not demonstrated. For overcoming this limitation, large population studies or meta-analysis will be needed. Secondly, when the neurological state of the patient was poor, we preferred to operate in the ICU. This selection bias could affect the mortality rate, as well as hospital and ICU day recorded.

From this study, we conclude that if aseptic protocols and precautions are kept strictly, ICU operations will not increase the risk of CNS infection and we expect that ICU operations could decrease the hospital and ICU days of surviving patients by saving neuro-critical time and avoiding unnecessary transfer of patients. As well as, neurosurgeons should keep in mind the serious outcomes of EVD-induced CNS infection and be cautious about the prevalence of MDR microorganisms as a pathogen of CNS infection.

\section{ORCID}

Si On Kim

http://orcid.org/0000-0001-5859-0830

\section{References}

1) Citerio G, Signorini L, Bronco A, Vargiolu A, Rota M, Latronico N; Infezioni LIquorali Catetere Correlate Study Investigators: External ventricular and lumbar drain device infections in ICU patients: a prospective multicenter Italian study. Crit Care Med 2015; 43: 1630-7.

2) Du B, Wang J, Zhong XL, Liang J, Xiang W, Chen D, et al: Single versus bilateral external ventricular drainage for intraventricular fibrinolysis using urokinase in severe ventricular haemorrhage. Brain Inj 2014; 28 : 1413-6.

3) Camacho EF, Boszczowski I, Freire MP, Pinto FC, Guimaraes T, Teixeira MJ, et al: Impact of an educational intervention implanted in a neurological intensive care unit on rates of infection related to external ventricular drains. PLoS One 2013 Feb 4 [Epub]. http://dx.doi. org/10.1371/journal.pone.0050708.

4) Edwards NC, Engelhart L, Casamento EM, McGirt MJ: Cost-consequence analysis of antibiotic-impregnated shunts and external ventricular drains in hydrocephalus. J Neurosurg 2015; 122: 139-47.

5) Foreman PM, Hendrix P, Griessenauer CJ, Schmalz PG, Harrigan MR: External ventricular drain placement in the intensive care unit versus operating room: evaluation of complications and accuracy. Clin Neurol Neurosurg 2015; 128: 94-100.

6) Cui Z, Wang B, Zhong Z, Sun Y, Sun Q, Yang G, et al: Impact of antibiotic- and silver-impregnated external ventricular drains on the risk of infections: a systematic review and meta-analysis. Am J Infect Control 2015; 43: e23-32.

7) Korinek AM, Reina M, Boch AL, Rivera AO, De Bels D, Puybasset L: Prevention of external ventricular drain-related ventriculitis. Acta Neurochir (Wien) 2005; 147: 39-45; discussion 45-6.

8) Ramanan M, Lipman J, Shorr A, Shankar A: A metaanalysis of ventriculostomy-associated cerebrospinal fluid infections. BMC Infect Dis 2015; 15: 3.

9) Gozal YM, Farley CW, Hanseman DJ, Harwell D, Magner M, Andaluz N, et al: Ventriculostomy-associated infection: a new, standardized reporting definition and institutional experience. Neurocrit Care 2014; 21: 147-51. 
10) Hetem DJ, Woerdeman PA, Bonten MJ, Ekkelenkamp MB: Relationship between bacterial colonization of external cerebrospinal fluid drains and secondary meningitis: a retrospective analysis of an 8-year period. $\mathrm{J}$ Neurosurg 2010; 113: 1309-13.

11) Scheithauer S, Bürgel U, Bickenbach J, Häfner H, Haase G, Waitschies B, et al: External ventricular and lumbar drainage-associated meningoventriculitis: prospective analysis of time-dependent infection rates and risk factor analysis. Infection 2010; 38: 205-9.

12) Lyke KE, Obasanjo OO, Williams MA, O'Brien M, Chotani R, Perl TM: Ventriculitis complicating use of intraventricular catheters in adult neurosurgical patients. Clin Infect Dis 2001; 33: 2028-33.

13) Conen A, Walti LN, Merlo A, Fluckiger U, Battegay M, Trampuz A: Characteristics and treatment outcome of cerebrospinal fluid shunt-associated infections in adults: a retrospective analysis over an 11-year period.
Clin Infect Dis 2008; 47: 73-82.

14) Ngo QN, Ranger A, Singh RN, Kornecki A, Seabrook JA, Fraser DD: External ventricular drains in pediatric patients. Pediatr Crit Care Med 2009; 10: 346-51.

15) Lo CH, Spelman D, Bailey M, Cooper DJ, Rosenfeld JV, Brecknell JE: External ventricular drain infections are independent of drain duration: an argument against elective revision. J Neurosurg 2007; 106: 378-83.

16) Scheithauer S, Bürgel U, Ryang YM, Haase G, Schiefer J, Koch S, et al: Prospective surveillance of drain associated meningitis/ventriculitis in a neurosurgery and neurological intensive care unit. J Neurol Neurosurg Psychiatry 2009; 80: 1381-5.

17) Schade RP, Schinkel J, Visser LG, Van Dijk JM, Voormolen JH, Kuijper EJ: Bacterial meningitis caused by the use of ventricular or lumbar cerebrospinal fluid catheters. J Neurosurg 2005; 102: 229-34. 